\title{
Plasmacytoid dendritic cells promote rotavirus-induced human and murine $B$ cell responses
}

\author{
Emily M. Deal, ${ }^{1}$ Katharina Lahl,2 Carlos F. Narváez, ${ }^{1,3}$ \\ Eugene C. Butcher, ${ }^{2,4,5}$ and Harry B. Greenberg'1,4,5,6

\begin{abstract}
1Department of Microbiology and Immunology and 2Department of Pathology, Stanford University School of Medicine, Stanford, California, USA. ${ }^{3}$ Program of Medicine, Universidad Surcolombiana, Neiva, Colombia. ${ }^{4}$ Veterans Affairs (VA) Palo Alto Health Care System, Palo Alto, California, USA. ${ }^{5}$ Palo Alto Institute for Research and Education, Palo Alto, California, USA.

${ }^{6}$ Department of Medicine, Stanford University School of Medicine, Stanford, California, USA.
\end{abstract}

\begin{abstract}
B cell-dependent immunity to rotavirus, an important intestinal pathogen, plays a significant role in viral clearance and protects against reinfection. Human in vitro and murine in vivo models of rotavirus infection were used to delineate the role of primary plasmacytoid DCs (pDCs) in initiating B cell responses. Human $\mathrm{pDCs}$ were necessary and sufficient for $\mathrm{B}$ cell activation induced by rotavirus. Type I IFN recognition by $\mathrm{B}$ cells was essential for rotavirus-mediated $B$ cell activation in vitro and murine $\mathrm{pDCs}$ and IFN- $\alpha / \beta-$ mediated $B$ cell activation after in vivo intestinal rotavirus infection. Furthermore, rotavirus-specific serum and mucosal antibody responses were defective in mice lacking functional pDCs at the time of infection. These data demonstrate that optimal $B$ cell activation and virus-specific antibody secretion following mucosal infection were a direct result of pDC-derived type I IFN. Importantly, viral shedding significantly increased in pDC-deficient mice, suggesting that $\mathrm{pDC}$-dependent antibody production influences viral clearance. Thus, mucosal $\mathrm{pDCs}$ critically influence the course of rotavirus infection through rotavirus recognition and subsequent IFN production and display powerful adjuvant properties to initiate and enhance humoral immunity.
\end{abstract}

\section{Introduction}

Humoral immunity provides a critical line of defense against microbial infection at mucosal surfaces. Within the intestine, the largest mucosal surface, secretory IgA is the most abundant antibody under steady-state conditions (1). T cell-dependent IgA induction occurs in response to many infectious agents and requires affinity maturation within germinal centers. Conversely, T cell-independent class switch leads primarily to production of low-affinity antibodies against commensal intestinal bacteria (2-5). Both mechanisms of IgA induction are critical to the maintenance of gut integrity, as mice deficient in the machinery of class switching to IgA or somatic hypermutation suffer from mucosal infections and hyperplasia in mesenteric LNs (MLN) and Peyer's patches $(6,7)$.

Plasmacytoid DCs (pDCs) are crucial first responders to viral infection due to their ability to rapidly induce type I IFN upon recognition of virally encoded molecular patterns, such as singlestranded RNA or DNA $(8,9)$. The resulting type I IFN response affects viral replication while simultaneously activating diverse immune effector cells (10). Specific ablation of pDCs during viral infection significantly diminishes the early type I IFN response (11-14). During herpes simplex-1 and vesicular stomatitis virus infection, pDC depletion impairs $\mathrm{CD}^{+} \mathrm{T}$ cell immunity $(12,15)$; similarly, during murine cytomegalovirus infection, the absence of pDCs results in diminished activation of early, nonspecific NK cells (12). pDCs can also activate B cells and induce antibody class switch through type I IFN (16-19). These studies, however,

Authorship note: Emily M. Deal and Katharina Lahl contributed equally to this work.

Conflict of interest: The authors have declared that no conflict of interest exists. Citation for this article: J Clin Invest. 2013;123(6):2464-2474. doi:10.1172/JCI60945. involved CD40 engagement on the B cell as a secondary stimulus. Microbiota-triggered type I IFN derived from intestinal stromal epithelial cells acts on pDCs to induce IgA during steady state (20). However, the mechanisms governing optimal induction of mucosal antigen-specific IgA responses to viral infection and the role of pDCs in this setting remain unclear.

We have recently demonstrated that stimulation of purified primary human pDCs with rotavirus (RV), an important intestinal pathogen in humans and many animals, directly induces secretion of cytokines, including large amounts of type I IFN (21). Additional studies demonstrate that lymphoid, but not epithelial, cells produce the majority of intestinal type I IFN after in vivo RV infection (22). As such, we sought to determine whether pDCs directly contribute to $\mathrm{B}$ cell activation and subsequent virus-specific antibody production, using both the human in vitro and murine in vivo models of RV infection. B cells represent an important component of the protective immune response against RV: mice deficient in $B$ cells are susceptible to reinfection as adults (23), and antibodies, but not $\mathrm{T}$ cells, mediate long-term protection from reinfection (24-27). Additionally, RV-specific serum antibodies are sufficient to suppress RV antigenemia in the absence of T cells (28), and transfer of B cells is sufficient to effect RV clearance in chronically infected Rag-knockout mice (29). Passive transfer of highly purified human IgM memory B cells into NOD/Shi-SCID IL-2R $\gamma^{\text {null }}$ immunodeficient mice suppresses systemic RV viremia, but not fecal shedding (30). In vivo RV infection results in early and substantial $\mathrm{B}$ cell activation (31-33), which is also observed following in vitro $\mathrm{RV}$ infection of human PBMCs (34). This activation requires the presence of accessory cells; purification of human B cells prior to infection completely abrogates this response (34). These effects are likely mediated by an innate cell, as comparable levels of B cell acti- 
A
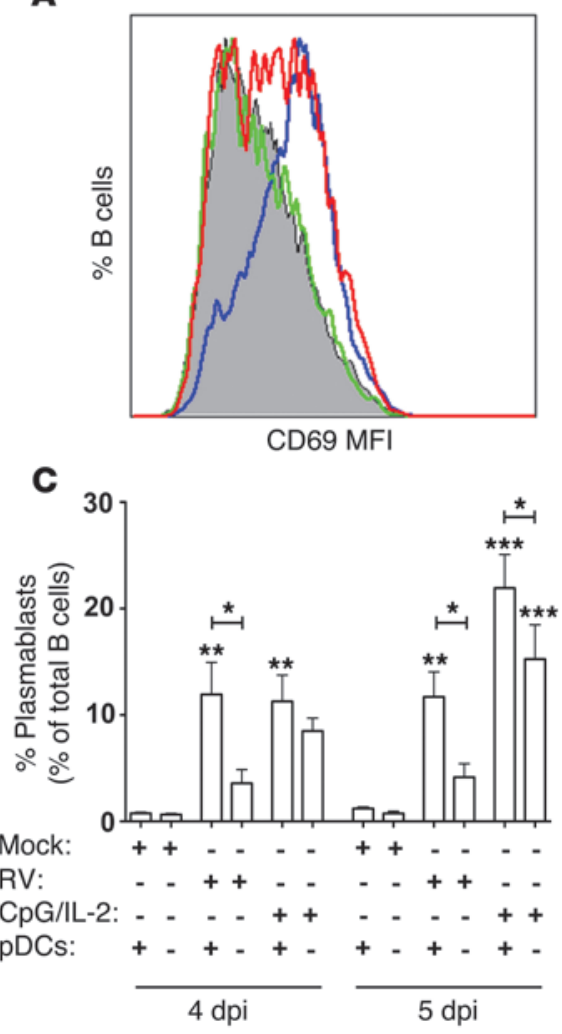

B

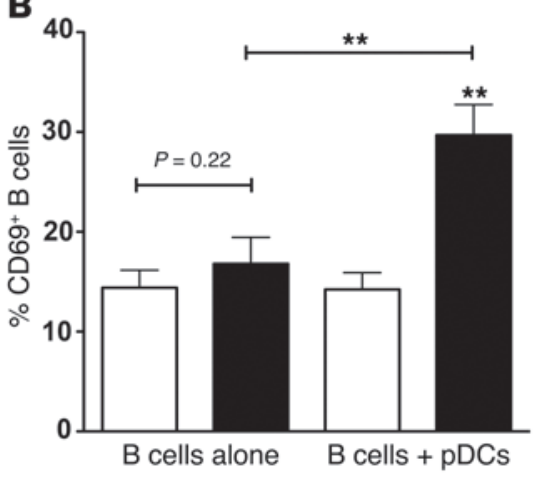

D

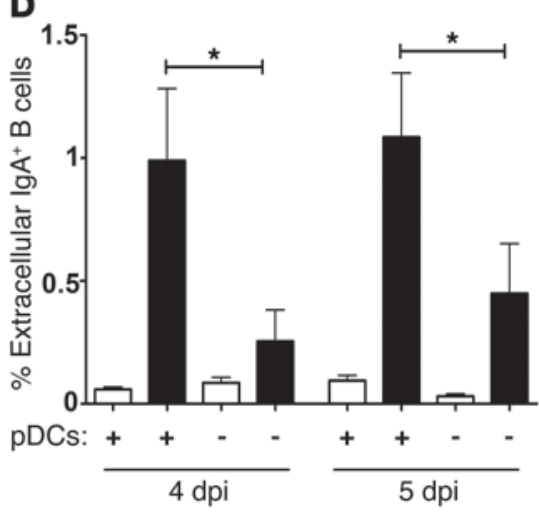

Figure 1

pDCs mediate human $B$ cell activation and plasmablast induction by RV in vitro. (A) The necessity of pDCs for the induction of CD69 expression on human $B$ cells within indicated PBMC preparations was assessed by flow cytometry 12 hours following mock (black) or RV stimulus. Blue, total PBMCs; green, pDC-depleted PBMCs; red, reconstituted pDC-depleted PBMCs. One experiment representative of 3 donors is shown. (B) The sufficiency of pDCs to induce $B$ cell activation by RV was assessed by flow cytometry for expression of CD69 by purified human B cells 12 hours following mock (white) or RV (black) stimulus in the presence or absence of pDCs, as indicated. ${ }^{* *} P=0.008$, Wilcoxon signed rank test; $n=9$. (C) The necessity of primary human $\mathrm{pDCs}$ to induce plasmablasts (CD3-CD19 ${ }^{+} \mathrm{CD} 20^{10} \mathrm{CD} 38^{+}$cells) was assessed by flow cytometry 4 and 5 days after the indicated stimulus. ${ }^{*} P \leq 0.05$; ${ }^{* *} P \leq 0.01$; ${ }^{* * *} P \leq 0.001$, repeated measures ANOVA with Neuman-Keuls multiple comparison test; $n=3$. (D) The percentage of B cells expressing extracellular $\lg \mathrm{A}$ at the indicated times after mock (white) or RV (black) stimulus. ${ }^{*} P<0.05$, paired $t$ test; $n=3$. vation are observed in wild-type and $\mathrm{T}$ cell receptor-deficient mice (31). We hypothesize that pDCs mediate RV-associated B cell activation and antigen-specific maturation and facilitate class switch, thus enhancing enteric clearance of RV infection.

\section{Results}

$p D C$ are necessary and sufficient for human $B$ cell activation by $R V$ in vitro. RV infection triggers early and substantial B cell activation in vivo (31). We have previously demonstrated that human B cells become activated, as evidenced by CD69 expression, following RV infection of total PBMCs and that this activation is abrogated upon B cell purification (34). Although pDCs perform several important antiviral functions in vivo, other cells, including macrophages and myeloid DCs (35), can also secrete type I IFN in response to infection. We first assessed whether $\mathrm{pDCs}$ are required for in vitro $B$ cell activation in response to RV. Human B cells within PBMC preparations depleted of $\mathrm{pDCs}$ failed to upregulate the early activation marker CD69 in response to in vitro RV stimulus (Figure 1A). The observed lack of B cell activation was not due to the selection procedure, as reconstitution of the cultures with purified autologous pDCs restored CD69 upregulation so that it was comparable to the levels reached in whole PBMC cultures (Figure 1A). Together, these experiments show that pDCs are necessary for $\mathrm{B}$ cell activation by $\mathrm{RV}$ in vitro.

To elucidate whether pDCs directly mediate B cell activation in response to RV, we purified human B cells from PBMCs and infected them with RV in the presence or absence of autologous pDCs. We assessed B cell activation, as measured by CD69 expression 12 hours after infection (Figure 1B). In agreement with previous reports (34), RV infection of purified B cells alone did not result in CD69 upregulation. In the presence of $\mathrm{pDCs}$, however, we observed a significant increase in the percentage of cells expressing CD69 (Figure 1B). This activation was only observed in the presence of both pDCs and RV, indicating the specificity of the response. We conclude that $\mathrm{pDCs}$ are sufficient for in vitro human B cell activation by RV.

We have previously shown that RV induces B cells within total PBMCs, but not in isolation, to differentiate into antibodysecreting cells (ASCs) (34). The failure of purified B cells to differentiate into ASCs suggested that other cells participate in this process (34). To determine whether $\mathrm{pDCs}$ were required, we assayed total PBMCs or PBMCs depleted of pDCs for plasmablast induction 4 and 5 days after exposure to RV or mock stimulus (Figure 1C). Compared with total PBMC cultures, the percentage of CD $3-\mathrm{CD} 19^{+} \mathrm{CD} 20^{\mathrm{lo}} \mathrm{CD} 38^{\text {hi }}$ plasmablasts was significantly decreased in total PBMC cultures depleted of pDCs both 4 and 5 days after RV stimulus $(P<0.05$; repeated measures ANOVA with Neuman-Keuls multiple comparison test). Although the percentage of B cells expressing extracellular IgA was significantly decreased in the absence of $\mathrm{pDCs}$ (Figure 1D; $P<0.05$; paired $t$ test), surface and intracellular $\operatorname{IgM}, \operatorname{IgA}$, and $\operatorname{IgG}$ staining among the plasma cells that did form after RV stimulus was similar to that seen on plasma cells within total PBMCs exposed to RV (data not shown), suggesting that the plasma cells that formed in the absence of pDCs retained full functionality. These data demonstrate that $\mathrm{pDCs}$ are also necessary for the induction of human plasmablasts following RV stimulus in vitro.

While B cells represent approximately $10 \%$ of the circulating PBMC population, pDCs are exceedingly rare, composing approximately $0.1 \%$ of total PBMCs (36). Additionally, pDCs predomi- 

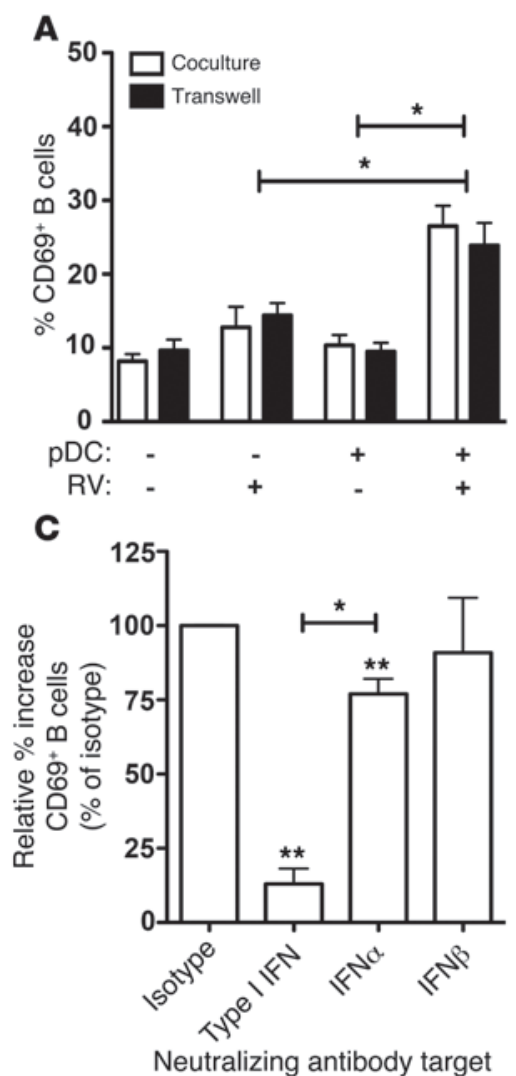

B

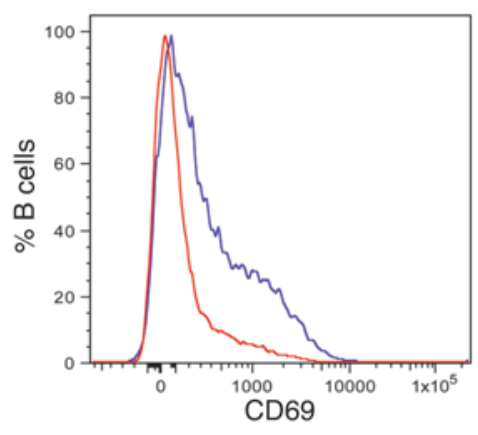

D

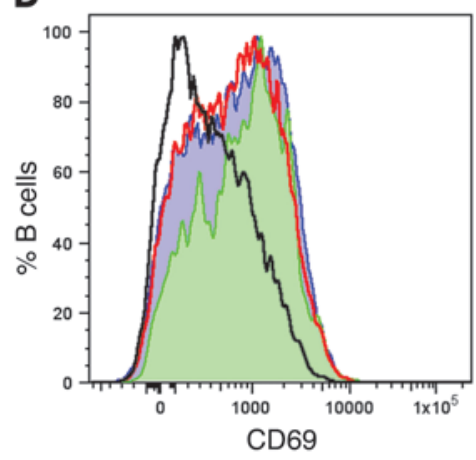

\section{Figure 2}

pDCs mediate human $B$ cell activation by soluble type I IFN. (A) B cell activation was assessed by flow cytometry analysis of CD69 expression by B cells cultured for 12 hours in the presence $(+)$ or absence $(-)$ of pDCs or RV as indicated. Cells were cocultured, allowing pDC/B cell contact (white), or with pDCs plated within Transwell inserts (black). ${ }^{*} P<0.05$, Mann-Whitney; $n=3-4$. (B) Representative histograms of CD69 expression by purified human B cells 12 hours after stimulus with 1:20 dilutions of supernatants from overnight cultures of pDCs exposed to mock (red) or RV (blue) stimulus. One experiment representative of 10 is presented. (C) CD69 expression was assessed by flow cytometry following overnight stimulus of purified B cells with supernatants from mock or RV-stimulated pDCs in the presence of the indicated neutralizing antibodies ( $\alpha$ IFN- $\alpha, \alpha$ IFN- $\beta$ or $\alpha$ IFN- $\alpha, \alpha$ IFN- $\beta$, and $\alpha$ IFN-receptor, indicated as "type I IFN"). The resulting increase in B cell activation is expressed as a percentage of that observed with the appropriate isotype control. ${ }^{* *} P=0.002$ vs. isotype, $\alpha$ IFN- $\alpha$ and $\alpha$ IFN- $\beta$, repeated measures ANOVA with Tukey's multiple comparison test; $n=3$. (D) CD69 expression by purified $\mathrm{B}$ cells following overnight stimulus with mock-stimulated pDC supernatant (black), RVstimulated pDC supernatant (green), RV-stimulated pDCs (blue), or IFN- $\alpha$ (red). Data depict 1 donor and are representative of 6 experiments. nately reside within the T cell-rich zones of the LN (37). Thus, in vitro coculture of $\mathrm{pDCs}$ and $\mathrm{B}$ cells may lead to $\mathrm{pDC}$ concentrations and cell-to-cell contact formations, greatly overrepresenting the frequency of these events in vivo. To this end, we sought to determine the minimal $\mathrm{pDC}$-to-B cell ratio required for $\mathrm{B}$ cell activation in vitro in response to $R V$ exposure. We titrated $\mathrm{pDCs}$ into $\mathrm{B}$ cell cultures at half-log increments and assessed CD69 expression after overnight culture when stimulated with RV or medium alone. These studies revealed that a very low $\mathrm{pDC} / \mathrm{B}$ cell ratio (0.0048), equating to approximately 1 pDC per $200 \mathrm{~B}$ cells, was sufficient to induce modest but significant increases in the percentage of $B$ cells expressing CD69 (Supplemental Figure 1; supplemental material available online with this article; doi:10.1172/JCI60945DS1). Activation was further increased and maximized when we cultured cells at a ratio of $1 \mathrm{pDC}$ per $42 \mathrm{~B}$ cells, which approximates the naturally occurring ratio observed in PBMCs (mean: 1 pDC per 30.3 B cells, $n=4$; Supplemental Figure 1 ). Thus, maximal pDCmediated $\mathrm{B}$ cell activation in vitro by $\mathrm{RV}$ occurs at physiologically relevant $\mathrm{PDC} / \mathrm{B}$ cell ratios.

pDCs mediate human B cell activation by soluble type I IFN. The observed sufficiency of low pDC-to-B cell ratios suggest that $B$ cell activation may depend on a soluble factor secreted by RVstimulated pDCs rather than on cell-to cell contact. To examine this hypothesis, we performed Transwell experiments in which we plated previously RV-exposed pDCs and B cells together or in separate chambers of the Transwell devices (Figure 2A). RV inoculation of $\mathrm{B}$ cells alone was insufficient to induce $\mathrm{B}$ cell activation. Likewise, the presence of pDCs in the absence of virus did not induce B cell activation. Importantly, RV-stimulated pDCs activated $\mathrm{B}$ cells regardless of whether $\mathrm{pDCs}$ were in direct contact with B cells or separated by the Transwell (Figure 2A). Thus, RVstimulated pDCs effect B cell activation through a soluble factor.

To confirm this observation, we harvested supernatants from pDCs following exposure to RV or mock stimulus. Supernatants from RV-stimulated, but not mock-stimulated, pDCs were sufficient to activate autologous or allogenic B cells following overnight culture (Figure 2B). Activation of B cells following addition of a 1:20 dilution of supernatant from RV-stimulated pDCs suggested that RV potently induced the factor that mediates human $B$ cell activation in pDCs.

\section{Table 1}

Cytokines and chemokines secreted by human pDCs following RV stimulus

\begin{tabular}{lc} 
Cytokine & pg/ml \\
IFN- $\alpha$ & 10,000 \\
IFN- $\beta$ & 7,000 \\
TNF- $\alpha$ & 1,300 \\
IL-6 & 200 \\
IL-8 & 2,000 \\
CXCL10 (IP-10) & 10,000 \\
CCL3 (MIP-1 $\alpha)$ & 2,000 \\
CCL4 (MIP-1 $\beta$ ) & 1,500 \\
CCL5 (RANTES) & 1,500 \\
\hline
\end{tabular}

Primary human pDCs secrete the indicated cytokines and chemokines within 12 hours of exposure to RV. Secreted CD40L does not increase in response to RV vs. mock stimulus. The following cytokines and chemokines are not detectable: BAFF, APRIL, IFN- $\gamma$, IL-12p40, IL-12p70, IL-1 $\beta$, IL-1RA, IL-2, or IL-4. Adapted from PLOS Pathogens (21). 
A

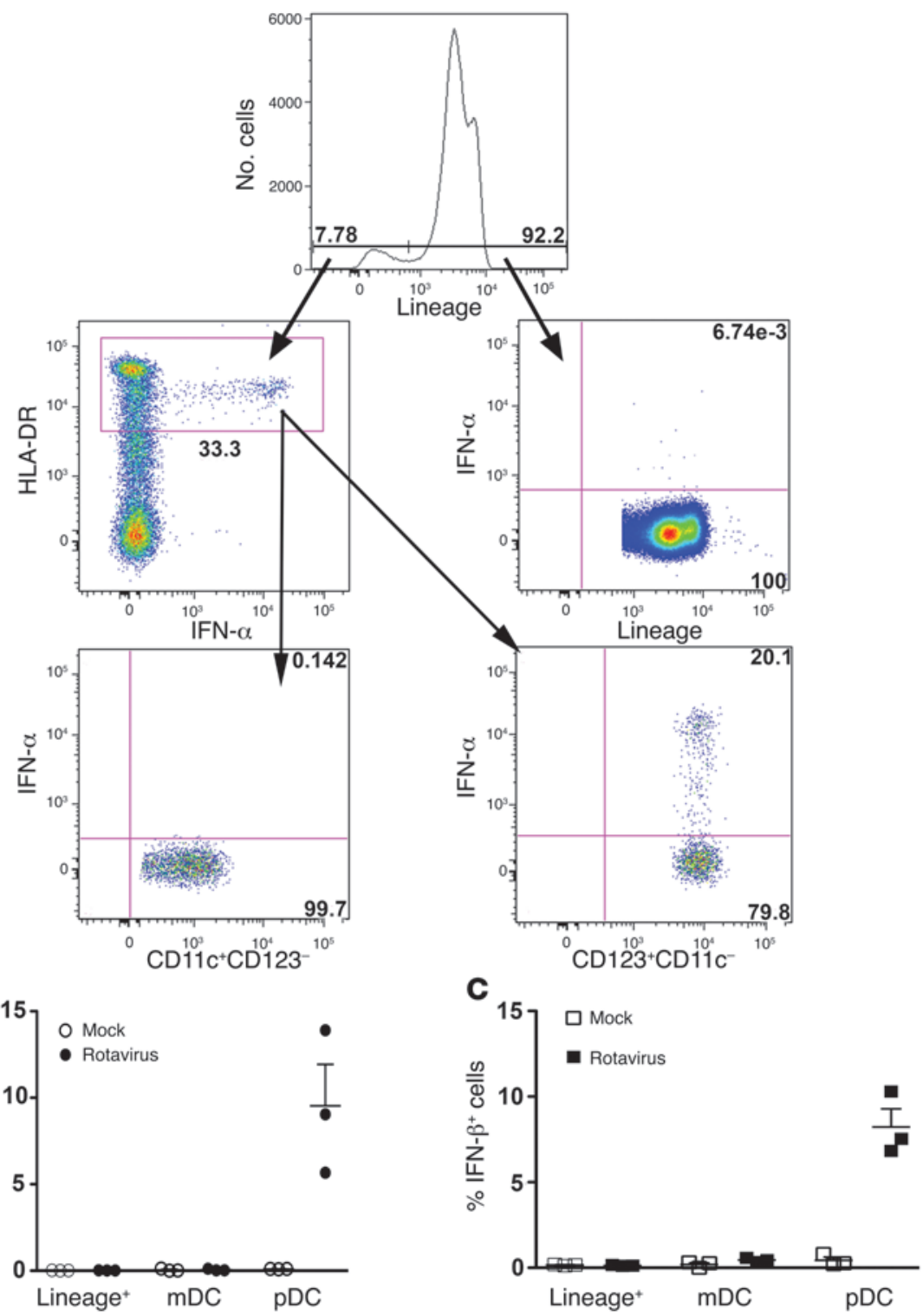

Figure 3

Identification of IFN- $\alpha^{+}$cells following RV infection of total PBMCs. (A) Intracellular staining for IFN- $\alpha$ was performed on total PBMCs 6 or 12 hours following RV infection. IFN- $\alpha$ was only observed in viable lineage HLA-DR+ cells, as indicated. Within this population, IFN- $\alpha$ staining was uniquely detectable within the $\mathrm{pDC}\left(\mathrm{CD} 123^{+} \mathrm{CD} 11 \mathrm{c}^{-}\right)$, but not myeloid $\mathrm{DC}$ $\left(\mathrm{CD} 123^{-} \mathrm{CD} 11 \mathrm{C}^{+}\right)$, population. Similar results are observed with IFN- $\beta ; n=3-4$. (B and C) Percentage IFN- $\alpha$-positive (B) or IFN- $\beta$-positive (C) cells in the indicated populations; $n=3$.

Although pDC-derived B cell-activating factor belonging to the TNF family (BAFF) and a proliferation-inducing ligand (APRIL) have been demonstrated to affect $\mathrm{B}$ cell responses, direct $\mathrm{pDC} / \mathrm{B}$ cell contact was required for this effect (20). We sought to determine whether RV induced BAFF or APRIL expression by pDCs and whether such expression was required to induce the $B$ cell activation phenotype. Approximately $5 \%$ of pDCs expressed detectable BAFF at 12 hours post RV infection (hpi), and we observed similar expression of APRIL (Supplemental Figure 2). However, neither BAFF nor APRIL was detectable in supernatants from RV-stimulated primary human pDCs at 6 or $12 \mathrm{hpi}$ (data not shown). Since cell contact is dispensable for $\mathrm{B}$ cell activation in our system (Figure 2A), these observations suggest that $\mathrm{pDCs}$ mediate the $\mathrm{B}$ cell response to $\mathrm{RV}$ through factors other than BAFF or APRIL.

pDCs secrete at least 10 cytokines and chemokines, including IFN- $\alpha$ and IFN- $\beta$, in response to RV stimulus (Table 1, adapted from ref. 21). Thus, we sought to determine whether one or more of these secreted factors was responsible for $\mathrm{B}$ cell activation by RV. We cultured purified B cells with supernatants from RV- or mock-stimulated pDCs in the presence or absence of neutralizing anti-cytokine/chemokine antibodies or the appropriate isotype controls. As illustrated in Figure 2C, CD69 expression by B cells exposed to supernatants from RV-simulated pDCs decreased significantly $(\sim 85 \%$; $P=0.002$, repeated measures ANOVA with Tukey's multiple comparison test) upon culture with a combination of antiIFN- $\alpha(\alpha$ IFN- $\alpha), \alpha$ IFN $-\beta$ and $\alpha$ IFN-AR2 (the receptor for type I IFN) neutralizing antibodies (indicated as type I IFN in Figure 2C). Incubation with $\alpha I F N-\alpha$ alone also significantly decreased the percentage of CD $69^{+} \mathrm{B}$ cells $(23 \%, P=0.002$, repeated measures ANOVA with Tukey's multiple comparison test) although to a far lesser extent $(P<0.05$, $\alpha$ Type 1 IFN vs. $\alpha$ IFN- $\alpha$ alone, repeated measures ANOVA with Tukey's multiple comparison test). Neutralization of IFN- $\beta$ alone resulted in a $10 \%$ (nonsignificant) decrease in the percentage of $\mathrm{CD} 69^{+} \mathrm{B}$ cells compared with cells treated with isotype controls. Importantly, maximal inhibition of $\mathrm{B}$ cell activation by $\mathrm{RV}$-stimulated $\mathrm{pDC}$ supernatants requires neutralization of both IFN- $\alpha$ and IFN- $\beta$ as well as neutralization of the type I IFN receptor, thus eliminating recognition of all type I IFNs by the B cell. While this demonstrates the partially redundant roles of IFN- $\alpha$ and IFN- $\beta$, it may also represent a contribution of IFN- $\varepsilon$, IFN- - , or IFN- $\omega$, minor type I IFN subtypes that also signal through IFN-AR. Culture with neutralizing $\alpha$ IL-6, $\alpha$ IL-8, $\alpha$ IP-10, $\alpha$ MIP- $1 \alpha, \alpha$ MIP-1 $\beta$, $\alpha$ RANTES, or $\alpha$ TNF- $\alpha$ did not significantly change the percentage of B cells activated (data not shown).

To determine whether IFN- $\alpha$, a major type I IFN subtype, was sufficient to induce $B$ cell activation or whether a minor type I IFN subtype was required, we incubated B cells with IFN- $\alpha$ in concentrations present in supernatants from RV-stimulated pDCs (Table 1, adapted from ref. 21). Both the percentage of B cells expressing CD69 and the MFI of CD69 on these B cells increased similarly to those of B cells exposed to supernatants from RV-stimulated pDCs or cultured directly with RV-stimulated pDCs (Figure 2D). We observed similar results following B cell stimulus with IFN- $\beta$ or IFN- $\omega$ (Supplemen- 
A

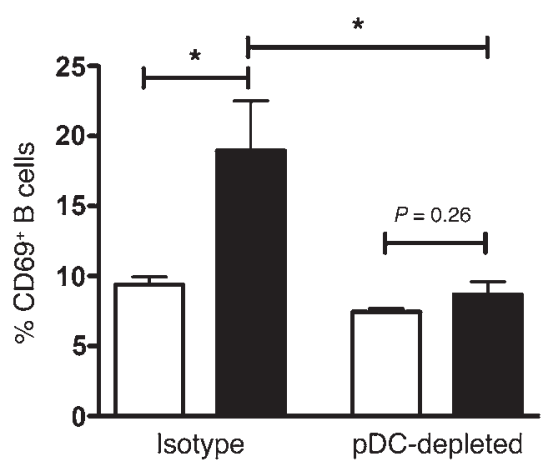

C

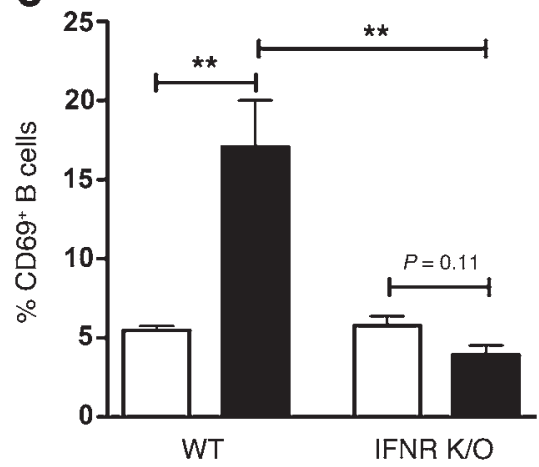

B

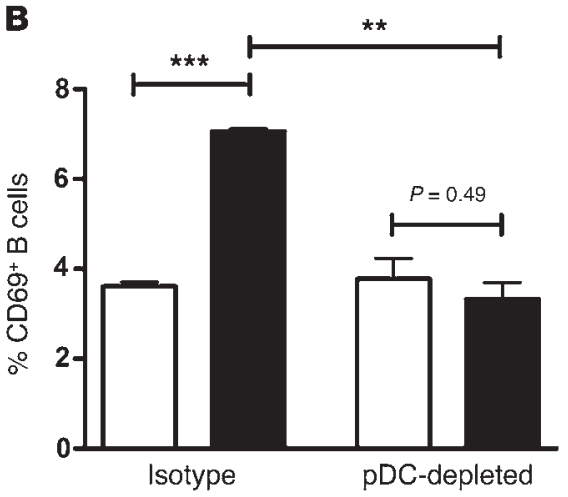

D

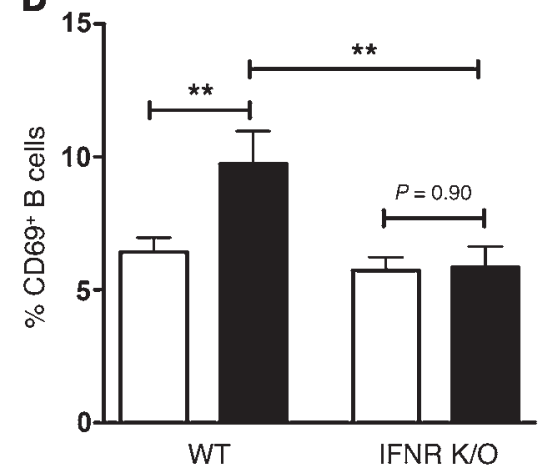

Figure 4

$\mathrm{pDC}$ and type I IFN signaling contribute to in vivo $B$ cell activation following murine RV infection. (A and B) Percentage of CD69+ B cells in the Peyer's patches (A) or MLN (B) of C57BL/6 mice depleted of $\mathrm{pDCs}$ or treated with isotype control 3 days after mock (white) or virulent murine RV (black) infection. (C and D) B cell activation in the Peyer's patches (C) and MLN (D) of wild-type SV129 or IFN-R knockout mice, 4 dpi. ${ }^{\star} P=0.03 ;{ }^{* \star} P=0.004 ;{ }^{* \star \star} P=0.0001, t$ test; $n=5$ per group.

tal Figure 3). Thus, all tested individual type I IFNs can mediate in vitro B cell activation. Importantly, intracellular staining of total PBMCs following RV stimulation revealed that production of IFN- $\alpha$ and IFN- $\beta$, the major type I IFN subtypes, was selectively observed in the pDC compartment (Figure 3).

$p D C$ and type I IFN signaling contribute to in vivo B cell activation following murine RV infection. We next sought to determine whether $\mathrm{pDCs}$ and/or type I IFN mediated B cell activation during $\mathrm{RV}$ infection in vivo. To this end, we depleted C57BL/6 mice of pDCs by i.p. injection with $\alpha$ PDCA-1 (aliases: BST2, CD317) antibody or treated with isotype control antibody, at days -3 and -1 prior to oral infection with wild-type murine RV (Supplemental Figure 4A). Although PDCA-1 is exclusively expressed by pDCs under steady-state conditions, other cell types, including $\mathrm{B}$ cells, upregulate this marker in response to type I IFN (38). Thus, we stopped $\alpha$ PDCA- 1 antibody treatment before RV infection in order to try to avoid inadvertent depletion of non-pDCs, such as activated B cells. Administration of anti- $\alpha$ PDCA-1 significantly reduced the frequency of $\mathrm{pDCs}$ in the gut-associated lymphoid tissue of mock- and RV-infected mice (Supplemental Figure 4, B-D), but did not affect the B cell population (Supplemental Figure 4E).

As previously reported (31), we observed significant increases in B cell activation in the Peyer's patches and MLN, but not spleen, of infected mice treated with isotype control antibody 3 days after RV infection (Figure 4, A and B). $\alpha$ PDCA- 1 treatment lacking functional pDCs early after infection. Whether the increased IgM reflects a deficit in class switching in the absence of pDCs or is a compensatory response to diminished IgG and IgA is unclear at this point. Importantly, total IgA, IgG, and IgM levels were similar among all groups of animals at 7 and $10 \mathrm{dpi}$ (data not shown), indicating that the defective response was restricted to virus-specific antibody production.

Intestinal $\operatorname{IgA}$ is an important component of the resolution of RV infection (39-41). As RV-specific antibody responses are observed in $\mathrm{T}$ cell receptor-deficient mice (27) and $\mathrm{CD}^{+} \mathrm{T}$ cells accelerate RV clearance $(23,42)$, we repeated our $\alpha$ PDCA- 1 antibody treatment regimen followed by oral RV infection in CD8deficient animals to assess the role of pDCs in the induction of $\mathrm{RV}$-specific fecal IgA during acute infection independent of the $\mathrm{CD}^{+} \mathrm{T}$ cell response. As observed in the serum, RV-specific fecal IgA production was significantly diminished following infection in $\alpha$ PDCA-1-treated CD8 knockout mice (Figure 6A).

To ascertain whether induction of the murine $\mathrm{B}$ cell response to RV specifically required pDC-derived type I IFN, we next employed an $\alpha$ SiglecH antibody, previously demonstrated to abrogate type I IFN production by pDCs in a DAP12-dependent manner (43). We treated wild-type C57BL6/J with $\alpha$ SiglecH antibody daily, from day -1 prior to infection through $5 \mathrm{dpi}$, as $\alpha$ SiglecH does not deplete pDCs (ref. 43 and Supplemental Figure 4). Consistent with the $\alpha$ PDCA-1-treated mouse results, a significant defect in B cell activation was also observed in the $\alpha$ SiglecH-treated mice (Supplemental Figure 5). This further 

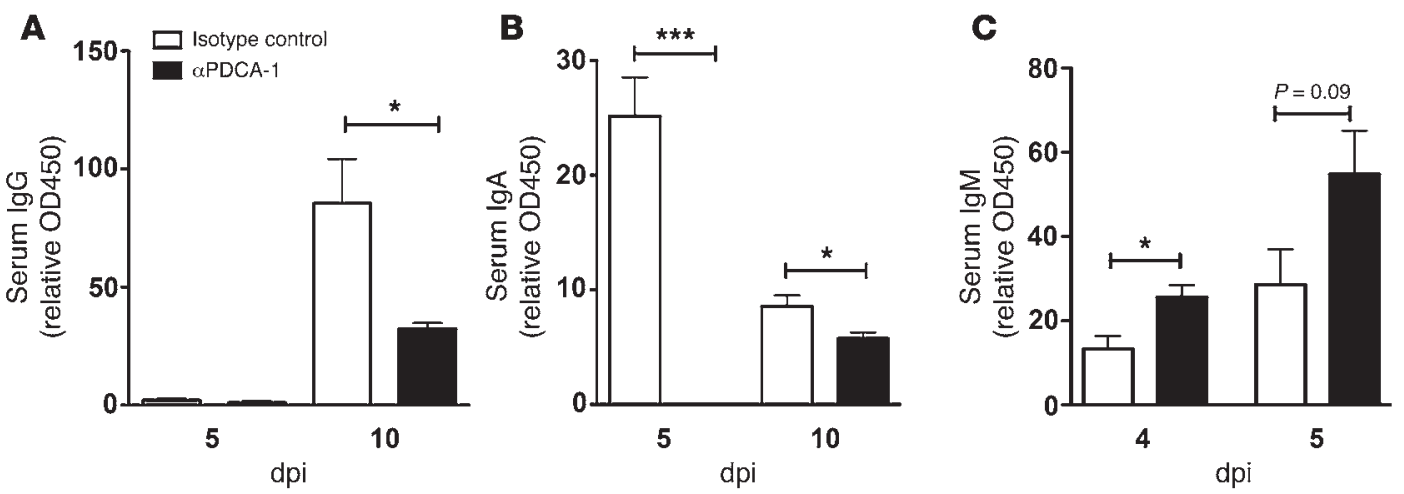

Figure 5

pDC-deficiency impairs in vivo serum antibody responses to RV. Serum RV-specific IgG (A), IgA (B), and IgM (C) were assessed by ELISA in isotype control (white) and PDCA-1-depleted (black) mice at the indicated dpi. ${ }^{\star} P \leq 0.05$; ${ }^{\star \star *} P \leq 0.001, t$ test; $n=5$ per group.

demonstrates that pDCs, through type I IFN, mediate B cell activation. Additionally, the deficiency in fecal RV-specific IgA was significantly more pronounced in $\alpha$ SiglecH-treated $\mathrm{CD}^{+} \mathrm{T}$ cell knockout mice (Figure 6A). To confirm these results, and to rule out possible nonspecific effects of the antibody treatment, we used BDCA2-DTR transgenic mice. pDC-depletion in these animals can be selectively induced by diphtheria toxin (DT) injection (12). Consistent with $\alpha$ PDCA-1- and $\alpha$ SiglecHtreated $\mathrm{CD}^{+} \mathrm{T}$ cell knockout mice, shedding of RV-specific IgA was significantly decreased in BDCA2-DTR animals following pDC depletion (Figure 6B). Since the BDCA2-DTR mice contain normal $\mathrm{CD}^{+} \mathrm{T}$ cell numbers, these data show a direct effect of pDCs that is not influenced by any possible side effects created by the lack of $\mathrm{CD}^{+} \mathrm{T}$ cells. Together, these data indicate that pDC-derived type I IFN is essential for the optimal induction of mucosal virus-specific antibody responses in vivo.

The defective $\mathrm{pDC}$ response enhances rotaviral shedding. We next examined the effects of impaired $\mathrm{PDC}$ responses on RV replication by examining fecal shedding of RV antigen in adult mice treated with selected anti-pDC antibodies (Figure 7A). As above, we used $\mathrm{CD} 8$ knockout mice in these studies, as $\mathrm{CD}^{+} \mathrm{T}$ cells themselves have been demonstrated to enhance RV clearance $(23,42)$. This approach enabled the direct examination of the pDC-instructed $\mathrm{B}$ cell response with regard to rotaviral clearance independent of cytotoxic T lymphocyte effects.

Both $\alpha$ PDCA-1- and $\alpha$ SiglecH-treated mice shed increased amounts of RV antigen in the feces compared with isotypecontrol animals following RV infection $(P<0.02$, Figure $7 \mathrm{~A})$. As expected, this was significantly more pronounced with the continual administration of the $\alpha$ SiglecH antibody compared with the animals treated with $\alpha$ PDCA- 1 antibody only prior to infection $(P=0.0011$, Figure 7A and Supplemental Figure 4). Peak viral shedding was observed in $\alpha$ SiglecH- and $\alpha$ PDCA-1-treated mice on $6 \mathrm{dpi}$, while maximal shedding was observed at $7 \mathrm{dpi}$ in the mice receiving the isotype control (Figure $7 \mathrm{~A})$. Furthermore, isotype

\section{Figure 6}

$\mathrm{pDC}$ deficiency impairs in vivo mucosal IgA responses to RV. Fecal IgA was assessed by ELISA in (A) isotype control (black), anti-PDCA-1treated (white), or anti-SiglecH-treated (gray) CD8 knockout or (B) Wild-type (black) or BDCA2-DTR (white) mice at the indicated dpi. ${ }^{\star} P \leq 0.05 ;{ }^{* *} P \leq 0.01{ }^{* * *} P \leq 0.001$, Mann-Whitney; $n=3-5$ per group. control-treated mice cleared virus by $8 \mathrm{dpi}$, at which time RV antigen was still detectable in the feces of $\alpha$ SiglecH- and $\alpha$ PDCA-1treated animals. Notably, total RV shedding was significantly increased in mice receiving $\alpha$ SiglecH $(P=0.0011$, Figure 7A). A similar statistically significant prolongation of shedding was observed in IFN-R knockout mice compared with SV129 wild-type mice ( $P=0.0152$, data not shown). Separate studies performed in BDCA2-DTR animals and their wild-type controls, which both had functional $\mathrm{CD}^{+} \mathrm{T}$ cells, revealed an early enhancement in viral shedding at $3 \mathrm{dpi}$ in the absence of pDCs (Figure 7B). We did not observe differences in viral clearance, presumably due to sufficiently strong $\mathrm{CD}^{+} \mathrm{T}$ cell responses in these mice (data not shown). Together, these data signify that the $\mathrm{pDC}$ response is integral to the early control of RV replication and contributes to viral clearance.
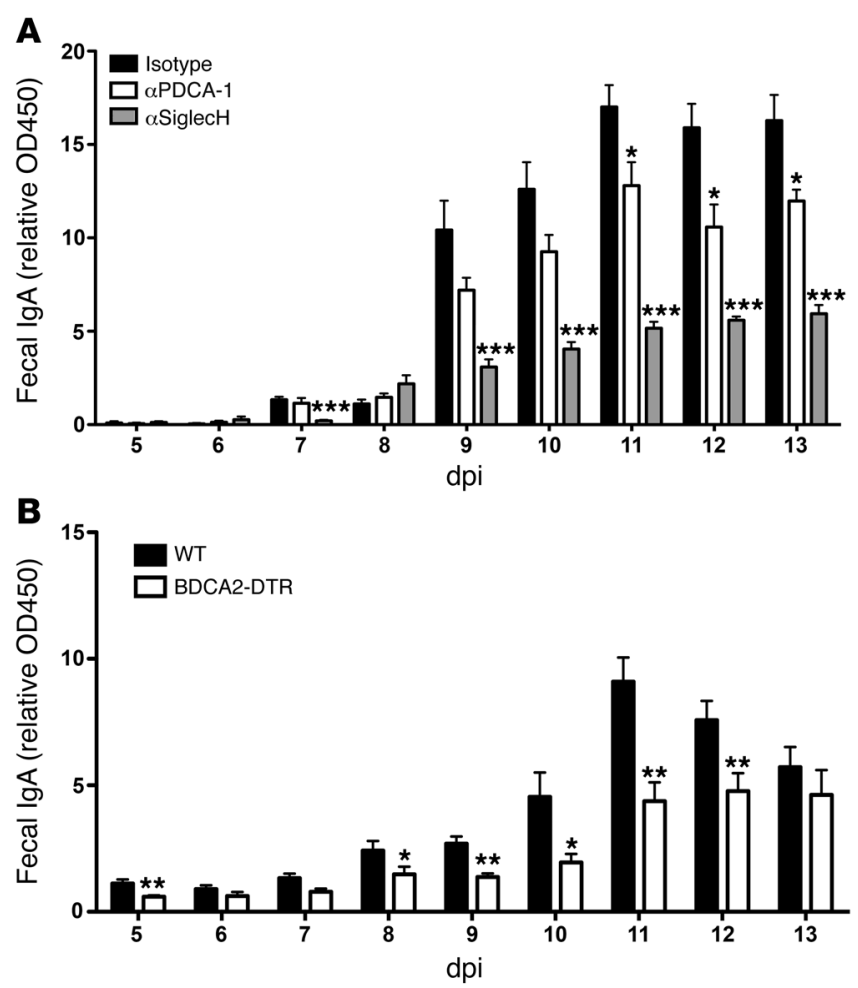


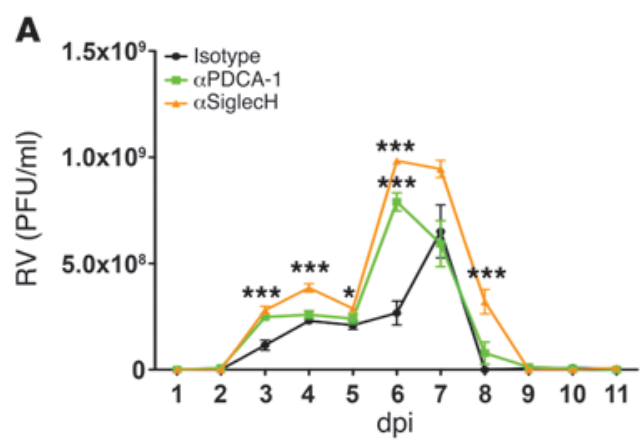

B

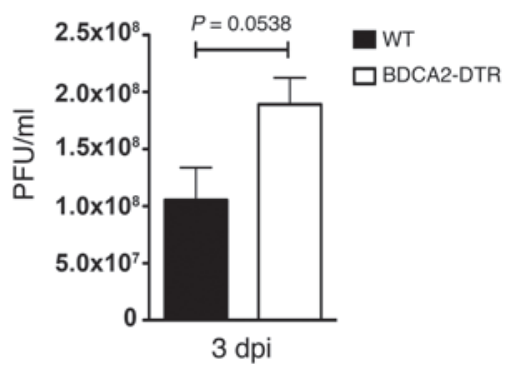

\section{Figure 7}

The defective $\mathrm{pDC}$ response enhances rotaviral shedding. (A) Fecal shedding of murine $\mathrm{RV}$ was assessed by ELISA in isotype control (black), anti-PDCA-1-treated (green) or anti-SiglecH-treated (orange) CD8 knockout mice at the indicated dpi. ${ }^{*} P<0.05$; ${ }^{* * *} P<0.001$, Mann-Whitney; $n=5$ per group. (B) Fecal shedding of murine RV 3 dpi in wild-type (black) or BDCA2-DTR (white) mice; Mann-Whitney.

\section{Discussion}

$B$ cells are critical for the generation of protective immunity to multiple viral infections, including RV. While transfer of either $\mathrm{T}$ or $\mathrm{B}$ cells to chronically infected Rag-knockout mice is sufficient to mediate $\mathrm{RV}$ clearance, suckling $\mathrm{J}_{\mathrm{H}} \mathrm{D}$ (B cell deficient) pups that cleared primary infection with murine RV were susceptible to reinfection as adults (23). Additionally, sporadic shedding has been reported in $\mathrm{J}_{\mathrm{H}} \mathrm{D}$ and $\mu \mathrm{MT}$ mice following initial viral clearance $(23,42)$. Consistent with these observations, B cell activation is observed in murine gut-associated lymphoid tissues, such as Peyer's patches and MLN, early following in vivo RV infection (31). This rapid B cell response is $\mathrm{T}$ cell independent in mice (32). Recent studies of human circulating and intestinal B cells stimulated with RV in vitro suggest that $B$ cell activation requires an accessory innate cell or molecule (34).

In the present study, we demonstrate that pDCs are necessary and sufficient for activation by RV in both in vitro and in vivo human and murine $\mathrm{B}$ cells, respectively. Consistent with previous descriptions of B cell activation during human cytomegalovirus infection (19), type I IFN mediates induction of B cell activation during RV infection. Importantly, and in contrast with previous findings utilizing influenza and human cytomegalovirus, the current study demonstrates that in vitro pDC-mediated early activation of B cells is independent of additional stimuli such as CD40 or B cell receptor ligation $(17,19,44)$.

Type I IFN has been demonstrated to upregulate expression of TLR7 and TLR9 by B cells (45-47), thus enhancing nucleic acid recognition and $\mathrm{B}$ cell responses. As pDC infection by RV is generally not productive (21) and transfer of supernatants alone from RV-stimulated pDCs is sufficient to induce B cell activation in the absence of virus (Figure 2B), direct recognition of $\mathrm{RV}$ by $\mathrm{B}$ cells appears dispensable for B cell activation in vitro. The present study does not exclude the possibility that recognition of type I IFN upregulates TLRs in the $\mathrm{B}$ cell, thus enhancing viral recognition and the $\mathrm{B}$ cell response. However, the sufficiency of $\mathrm{pDC}$ conditioned supernatants to induce $\mathrm{B}$ cell activation demonstrates that viral recognition by the $\mathrm{B}$ cell is not required for this initial activation.

Previous reports demonstrated that type I IFN mediates activation of LN-resident B cells in response to influenza or West Nile virus infection (48-51). In the context of West Nile virus infection, the authors excluded macrophages as type I IFN-producing cells, but the actual cellular source was not identified for either infection model. Here, we identify pDCs as the sole type I IFN- $\alpha / \beta$-producing cells in human PBMC cultures following RV stimulus (Figure 3). Furthermore, recent studies demonstrate that murine RV infection induces type I IFN production in lymphoid, but not epithelial, cells, bolstering the current findings (22). We have previously shown that primary human pDCs secrete approximately $10,000 \mathrm{pg} / \mathrm{ml}$ of both IFN- $\alpha$ and IFN- $\beta$ when cultured for $6-12$ hours at $1 \times 10^{6} \mathrm{pDCs} / \mathrm{ml}$ after RV stimulus (21). While the minor type I IFN- $\omega$ can induce $B$ cell activation (Supplemental Figure 3), the magnitude of the IFN- $\alpha / \beta$ response, combined with a specific decrease in $\mathrm{B}$ cell activation after IFN- $\alpha$ neutralization, implies a minor role for other type I IFN subtypes in this process.

To address pDC-mediated B cell activation in vivo as well as to examine the resulting impact on viral clearance, we employed the mouse model of homologous murine RV infection $(41,52)$. The absence of pDCs or the defect in the ability of pDCs to effect type I IFN secretion abolished mucosal B cell activation in vivo (Figure 4 and Supplemental Figure 5).

Transient administration of $\alpha$ PDCA-1, as carried out here, only temporarily ablates pDCs (Supplemental Figure 4). Since other cell types, including B cells, upregulate PDCA- 1 in response to type I IFN (38), we administered depleting antibody only at days -3 and -1 prior to infection, as previously described (11), to avoid the inadvertent depletion of other cell types that might have upregulated PDCA-1 following an IFN response to RV infection. To further

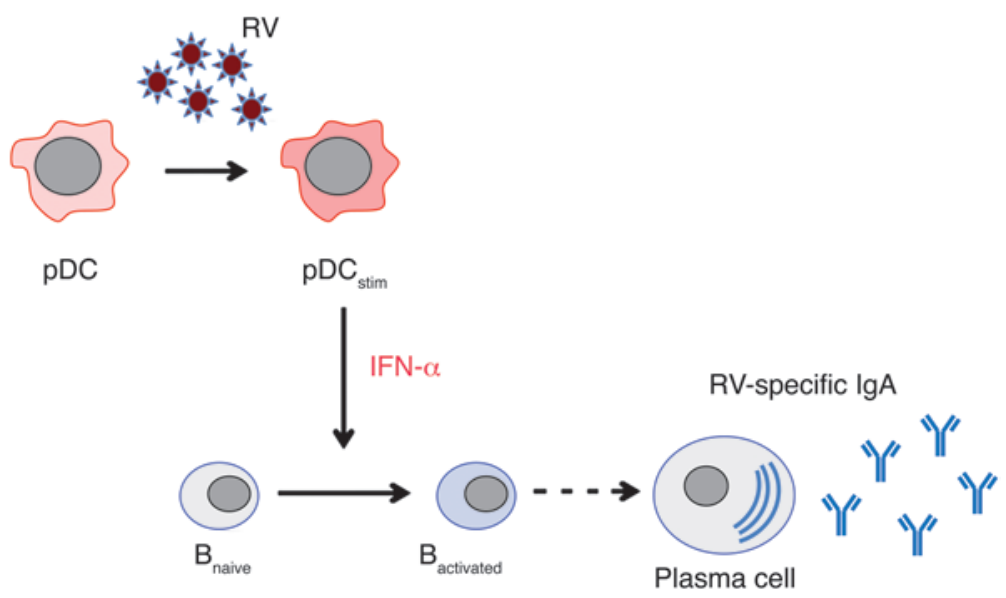

Figure 8

Model of B cell activation in response to RV. Immature pDCs sense RV in vivo and in vitro, leading to type I IFN production by pDCs. pDC-derived type I IFN activates naive $B$ cells, ultimately leading to the production of RV-specific IgA in the intestines. 
control for a specific effect of pDC depletion on B cell function in response to RV, we employed BDCA2-DTR mice. BDCA2 is a highly specific pDC marker in the human system. The mice we used here carry a transgene consisting of the human BDCA2 promoter coupled to the primate DT receptor. Previous studies have shown that DT injection faithfully depletes only pDCs in this model (12). The use of this alternative approach allows full ablation of the $\mathrm{pDC}$ response during the whole time course of infection, since we continually administered DT during the ongoing rotaviral infection and immune response. Additionally, the DTmediated depletion rules out potential nonspecific effects caused by depleting antibodies. In addition to demonstrating the requirements for pDCs through these complementary methods of pDC depletion, we also probed the role of pDC-dependent type I IFN production using a nondepleting $\alpha$ SiglecH antibody; engagement of SiglecH, a lectin receptor specifically expressed by pDCs and a very small subset of tissue-resident macrophages under steadystate and inflammatory conditions, specifically inhibits type I IFN production by pDCs (refs. 43, 53, and Supplemental Figure 6). Importantly, macrophages within the spleen and gut-associated lymphoid tissues did not costain with $\alpha$ SiglecH antibody by flow cytometry in our hands (data not shown). SiglecH engagement inhibited B cell activation and RV-specific fecal IgA production in our studies, strengthening our human in vitro data in suggesting a crucial role of type IFN in pDC-mediated B cell activation.

Downstream of early activation, we observed defective plasmablast formation in vitro as well as defects in antigen-specific serum and mucosal antibody responses in vivo in the absence of pDC activity (Figures 1, 5, and 6). The observed decrease in RV-specific serum IgG and IgA and accompanying increase in serum IgM in the absence of a functional $\mathrm{pDC}$ response suggest that $\mathrm{pDCs}$ may have a role in the induction of class switch after infection. It is important to note that we detected differences only in RV-specific, but not total, antibody levels, in the $\alpha$ SiglecH- and $\alpha$ PDCA-1treated animals. Although neither RV clearance nor protection from reinfection requires $\operatorname{IgA}(29,54,55), \operatorname{IgA}$ antibodies against $\mathrm{RV}$ structural proteins alone can mediate protection. IgA, but not IgG, monoclonal antibodies administered by a hybridoma "backpack" tumor are sufficient to protect naive newborn mice from primary infection (56), while human RV-specific IgA as well as IgG antibodies are able to neutralize RV in cell culture (25). Additionally, RV-specific IgA but not IgG can confer resistance to infection by neutralizing virus intracellularly following transcytosis $(40,57,58)$, and RV-specific serum IgA levels predict protection against severe clinical disease (59).

Our data provide, to the best of our knowledge, the first demonstration that pDCs, as well as pDC-derived type I IFN, contribute to the induction of virus-specific mucosal IgA antibody production and that the absence of this contribution impedes the resolution of RV infection. Although we did not address the effect of pDC-mediated B cell activation on later stages of functional B cell responses in this study, type I IFN has previously been implicated in antibody production at multiple stages, including modulation of plasma cell formation and antibody class switching $(17,49,60)$. Additionally, type I IFN is required for the formation of germinal centers in adenovirus infection (61).

While a recent study has demonstrated that pDCs modulate mucosal IgA production in the steady state (20), this was shown to require recognition of $\mathrm{pDC}$-derived BAFF or APRIL by B cells through direct $\mathrm{pDC} / \mathrm{B}$ cell contact. Importantly, production of type I
IFN by stromal cells was essential for the induction of BAFF and APRIL expression by pDCs (20). Our findings extend these steadystate observations, as the present data demonstrate that viral stimulus of pDCs overcomes the need for extrinsic epithelial cellderived type I IFN for induction of antigen-specific IgA responses. As pDCs rapidly secrete large quantities of type I IFN in response to RV stimulus (21), we performed in vitro studies to examine whether such stimulus was sufficient for the induction of BAFF or APRIL expression by purified human pDCs. The demonstration that RV stimulus induces BAFF and APRIL production, but not secretion, by pDCs (Supplemental Figure 2 and data not shown) combined with the dispensability for $\mathrm{PDC} / \mathrm{B}$ cell contact for the induction of the $\mathrm{B}$ cell response (Figure $2 \mathrm{~A}$ ) indicates that these cytokines are not likely to contribute to the observed in vitro B cell activation. The induction of BAFF and APRIL by pDC-derived type I IFN, independent of stromal cells, would ensure the availability of these ligands for recognition by $\mathrm{B}$ cells and thus allow for their contribution to the induction of the mucosal IgA response. As Peyer's patch-resident pDCs do not efficiently secrete type I IFN (62), we hypothesize that $\mathrm{pDCs}$ within the lamina propria or MLN are critical for the induction of the $\mathrm{B}$ cell response. Previous studies in suckling mice demonstrated the presence of RV within MLNs during acute infection (63). Furthermore, it has been demonstrated that lymphoid, but not epithelial, intestinal cells produce type I IFN in response to $\mathrm{RV}$ infection (22). Studies are currently underway to further elucidate the mechanisms governing this process.

The induction of an anti-RV antibody response can mediate viral clearance and is critically responsible for preventing reinfection (23). The rapid initiation of the pDC type I IFN response by RV appears to accelerate this process as part of the host defense mechanism (Figure 7). This is evidenced by increased viral shedding early after infection of $\alpha$ PDCA-1- or $\alpha$ SiglecH-treated mice as well as in the BDCA2-DTR model. Although $\mathrm{CD}^{+} \mathrm{T}$ cells enhance the timely clearance of rotaviral infection and $\mathrm{T}$ cells alone can mediate short-term partial protection against RV, RV-induced early B cell activation is T cell independent (31). We thus assessed the role of $\mathrm{pDC}$-mediated $\mathrm{B}$ cell responses in the absence of $\mathrm{CD}^{+}$ $\mathrm{T}$ cells. As such, we were able to eliminate the role of cytotoxic $\mathrm{T}$ lymphocyte responses in the induction of mucosal antibody production and, importantly, in primary viral clearance (Figure 6A and Figure 7A). Of note, however, we also saw significant defects in RV-specific fecal IgA and, to a lesser degree, in viral shedding in pDC-deficient BDCA2-DTR animals with an intact $\mathrm{CD}^{+} \mathrm{T}$ cell pool (Figure 6B and Figure 7B). Restoration of the $\mathrm{pDC}$ population after transient $\alpha$ PDCA-1-mediated depletion (Supplemental Figure 4) may have contributed to the more rapid clearance of $\mathrm{RV}$ infection and prevented viral shedding and replication from reaching the magnitude observed in $\alpha$ SiglecH-treated animals. Similarly, the intact $\mathrm{CD}^{+} \mathrm{T}$ cell response in BDCA2-DTR mice likely contributed to the timely resolution of RV shedding in these mice, thus causing kinetics of clearance similar to those observed in wild-type animals (Figure 7B and data not shown). Importantly, defects in the RV-specific fecal IgA response were observed in the CD8-replete BDCA2-DTR animals as they were in the $\alpha$ PDCA and $\alpha$ SiglecH-treated mice (Figure $6 \mathrm{~B}$ ), thus supporting the critical role of $\mathrm{pDCs}$ in this local IgA response.

To the best of our knowledge, the current work demonstrates for the first time the critical requirement of pDC-derived type I IFN for the initiation of both $\mathrm{B}$ cell activation and the optimal initiation of mucosal and systemic antigen-specific antibody responses to a 
viral infection. Importantly, our studies demonstrate that pDCs stimulated with RV have powerful adjuvant properties for B cell activation in vitro and in vivo. In our mouse model of homologous murine RV infection, this allows for potent antibody production and influences viral clearance efficiency. Given the lack of functional differences in response to other systemically administered viruses in $\mathrm{pDC}$-deficient models, we suggest that our findings on the role of $\mathrm{pDCs}$ in the modulation of $\mathrm{B}$ cell responses, summarized in Figure 8, may be specifically relevant to mucosal tissues. We have not investigated whether $\mathrm{pDC}$ ablation has consequences beyond the $\mathrm{B}$ cell response or whether murine $\mathrm{pDCs}$ vary in their response to homologous versus heterologous (nonmurine) RV strains. We chose to use a homologous murine RV model because it was only with such a model that the modulating effects on viral replication by $\mathrm{pDCs}$ could be fully assessed. If the effects of $\mathrm{pDCs}$ on $\mathrm{B}$ cell responses are conserved during heterologous infection, type I IFN production by pDCs exposed to future heterologous $\mathrm{RV}$ vaccine candidates may serve as a predictive marker to measure their capacity to induce an optimal adaptive immune response in the gut. Since the humoral immune response at mucosal sites is a critical defense barrier for many opportunistic infections, pDCmediated $\mathrm{B}$ cell activation may be a key component of protective immunity to mucosal pathogens.

\section{Methods}

Virus preparation and inactivation. Simian tissue-culture adapted RV (RRV) was grown in fetal monkey kidney (MA104) cells in the presence of tryp$\sin$ as previously described $(64,65)$. Virus was trypsin activated $(5 \mu \mathrm{g} / \mathrm{ml})$ at $37^{\circ} \mathrm{C}$ for $20-30$ minutes prior to $\mathrm{pDC}$ or $\mathrm{B}$ cell infection. All preparations were endotoxin-free, as determined by Limulus amebocyte lysate test (Charles River). RRV preparations were titrated by plaque assay on MA104 cells and expressed as number of PFU/ml, as described (65). The virulent wild-type, non-cell-culture-adapted murine $\mathrm{RV}$ strain $\mathrm{EC}_{\mathrm{w}}$ was used to infect mice. This virus causes diarrhea in $100 \%$ of 5 -day-old suckling mice, which lasts for approximately 7-9 days (52). The virus was propagated in suckling mice, used as a clarified intestinal homogenate, and its 50\% diarrhea dose $\left(\mathrm{DD}_{50}\right)$ was determined as previously described (52).

Isolation of primary buman $p D C$ and B cells. PBMCs were isolated from anonymized leukoreduction chambers obtained from the Stanford Blood Center by centrifugation over Ficoll-Hypaque (GE Healthcare). pDCs were negatively selected using the pDC Isolation Kit (Miltenyi Biotec), according to the manufacturer's instructions. To increase the purity of the pDC preparations, consecutive purifications were performed using an AutoMACS Pro (Miltenyi Biotec). An average of $2 \times 10^{6} \mathrm{pDCs}$, defined as viable, lineage HLA-DR ${ }^{+} \mathrm{CD} 11 \mathrm{c}^{-} \mathrm{CD} 123^{+}$cells, were isolated per donor; preparations were routinely more than $85 \%$ pure. B cells were negatively selected using the human B cell Isolation Kit II and AutoMACS Pro Separator (Miltenyi Biotec), according to the manufacturer's instructions.

Depletion of $p D C$ from total human PBMCs. To ensure complete depletion of pDCs from human PBMCs, pDCs were positively selected by consecutive labeling with CD304 (BDCA4/Neuropilin-1) and CD303 (BDCA2) human microbead kits (Miltenyi Biotec) according to the manufacturer's instructions. Separation was performed using an AutoMACS Pro Separator. This resulted in more than $98 \%$ depletion of the $\mathrm{pDC}$ population, as determined by flow cytometry.

Human PDC culture, infection, stimulation, and blocking studies. Primary human pDCs, B cells, or total PBMCs were exposed to RRV or mock stimulus at a multiplicity of infection of 5 for 1 hour in serum-free RPMI 1640 (CellGro) supplemented with penicillin/streptomycin and L-glutamine (Gibco; Invitrogen). pDCs were present or absent in the B cell and PBMC infections, as indicated. Cells were washed and subsequently cultured at a concentration of $1 \times 10^{6} \mathrm{cells} / \mathrm{ml}$ in RPMI 1640 with $10 \%$ heat-inactivated fetal bovine serum (Hyclone), penicillin/streptomycin, and L-glutamine until harvest.

Human pDC and B cell Transwell cultures. pDCs and B cells were isolated from PBMCs as described above. Each cell type was exposed to RRV (multiplicity of infection 5) in isolation for 1 hour in serum-free medium prior to plating. Cells were washed and resuspended at a concentration of $1 \times 10^{6}$ cells $/ \mathrm{ml}$ in RPMI 1640 with $10 \%$ heat-inactivated fetal bovine serum, penicillin/streptomycin, and L-glutamine. pDCs and B cells were cultured in 12-well plates in the presence or absence of 0.4- $\mu \mathrm{m}$ Transwell inserts (Corning Inc.) at a ratio of 0.4 pDCs per B cell. When applicable, pDCs were plated within the Transwell and B cells in the well.

Cytokine neutralization and stimulus. B cells were cultured with pDC-conditioned supernatants in the presence or absence of neutralizing polyclonal antibodies against IFN- $\alpha$, IFN- $\beta$, IFN-AR2 (PBL Interferon Source), MIP-1 $\alpha$, MIP-1 $\beta$, IP-10, RANTES, TNF- $\alpha$, IL-6, IL-8, or the isotype controls, normal goat IgG (R\&D Systems Inc.), or normal sheep serum (Jackson ImmunoResearch Laboratories) at a concentration of $1 \times 10^{6} \mathrm{~B}$ cells/ml in RPMI 1640 with $10 \%$ heat-inactivated fetal bovine serum, penicillin/streptomycin, and L-glutamine overnight until harvest. Where indicated, purified B cells were cultured with IFN- $\beta$ or IFN- $\omega$ (Pestka Biomedical Laboratories Inc.).

Plasmablast induction. Total human PBMCs or those depleted of pDCs were cultured for 4 or 5 days in 12 -well plates at a concentration of $2 \times 10^{6}$ cells $/ \mathrm{ml}$ in RPMI 1640 with $10 \%$ heat-inactivated fetal bovine serum, penicillin/streptomycin, and L-glutamine after exposure to RRV or mock stimulus, as described above. CpG 2006 (3 $\mu \mathrm{g} / \mathrm{ml}$; Invivogen) and IL-2 $(10 \mathrm{ng} / \mathrm{ml})$ were added to mock-stimulated cultures, as indicated, as a positive control for plasmablast induction. After culture, cells were washed and stained for analysis by flow cytometry.

Flow cytometry (FACS). Supernatants were harvested by centrifugation of cultured pDCs, B cells, or PBMCs, which were then washed once with PBS (CellGro). The LIVE/DEAD Aqua Dead Cell Stain Kit (Invitrogen) was utilized to assess cellular viability via amine exclusion. Surface staining was performed using antibodies against human CD3, CD14, CD16, CD27, CD38, HLA-DR, CD69, CD123 (BD Biosciences), CD11c, CD19, CD20 (eBioscience), and BDCA2 and BDCA4 (Miltenyi Biotec); $\mathrm{pDCs}$ were defined as being lineage HLA-DR ${ }^{+} \mathrm{CD} 11 \mathrm{c}^{-} \mathrm{CD} 123^{+}$ and costained with BDCA2 and BDCA4 (21). Plasmablasts were defined as $\mathrm{CD} 3{ }^{-} \mathrm{CD} 19^{+} \mathrm{CD} 20^{\text {lo }} \mathrm{CD} 38^{\text {hi }}$ cells (66). Cellular fixation and permeabilization were performed using Cytofix/Cytoperm (BD Biosciences) per the manufacturer's instructions prior to intracellular staining for IFN- $\alpha$ (BD Biosciences), IFN- $\beta$ (Antigenix America), BAFF and APRIL (R\&D Systems Inc.), IgM, IgG (Jackson ImmunoResearch Laboratories Inc.), or IgA (Miltenyi Biotec). When appropriate, surface immunoglobulins were blocked prior to permeabilization using $\mathrm{F}\left(\mathrm{ab}^{\prime}\right) 2$ goat anti-human Igs (Jackson ImmunoResearch Laboratories Inc.). Murine lymphocytes isolated from spleens, Peyer's patches, or MLNs were stained using antibodies against murine CD4, CD8, CD19, CD69, B220 (BD Biosciences), and SiglecH (eBioscience).

Data were acquired using a LSRII cytometer and DIVA software (BD Biosciences); analysis was performed using FlowJo (Treestar Inc).

Detection of secreted cytokines and chemokines. Supernatants of cultures more than $85 \%$ (mean \pm SEM: $90.84 \% \pm 0.7499$ ) pure for pDCs were analyzed by ELISA (R\&D Systems Inc.) per the manufacturer's instructions for the presence of BAFF or APRIL. Additional cytokine and chemokine secretion was determined by Luminex using MILLIPLEX MAP (Millipore) or ELISA (Pestka Biomedical Laboratories Inc.) as previously described (21). Murine IFN- $\alpha$ was captured using the monoclonal antibodies RMMA-1 (Pestka Biomedical Laboratories) and F18 (Hycult Biotech Inc.) and detected with polyclonal rabbit $\alpha$ IFN- $\alpha$ (Pestka Biomedical Laboratories Inc). 


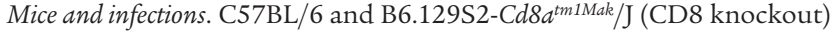
mice were purchased from The Jackson Laboratory. IFN-R knockout and wild-type 129Sv/Ev were obtained from B\&K Universal Ltd. BDCA2-DTR mice were provided by Marco Colonna (Washington University, Saint Louis, Missouri, USA). All mice were maintained at the Palo Alto VA Health Care System Veterinarian Medicine Unit. Four- to six-week-old adult mice were orally inoculated with $3 \times 10^{3} \mathrm{DD}_{50}$ of $\mathrm{EC}_{\mathrm{w}}$ diluted in M199 medium (Gibco; Invitrogen) or M199 medium alone. Mice were sacrificed at the indicated dpi, and serum, Peyer's patches, MLN, and spleen were collected and lymphocytes isolated as previously described (67). Lymphocytes were maintained in RPMI 1640 with 10\% heat-inactivated fetal bovine serum, penicillin/streptomycin, and L-glutamine prior to analysis by flow cytometry.

In vivo pDC depletions. C57BL/6 mice were injected i.p. with $100 \mu \mathrm{g}$ functional-grade purified anti-PDCA-1 (Miltenyi Biotec), anti-SiglecH (AbD Serotec), or rat IgG2b isotype control (BioLegend) as previously described $(11,12)$. Alternatively, BDCA2-DTR mice were injected i.p. with $200 \mathrm{ng}$ DT (Merck) at days $-2,-1$, and 0 prior to infection and 200 ng every 2 dpi (12).

ELISA. RV-specific fecal or serum antibody was detected by ELISA as previously described (23), using peroxidase-labeled rat anti-mouse IgM (Southern Biotech) or goat anti-mouse IgA or IgG (Kirkegaard \& Perry Laboratories). Total IgA and IgG concentrations were determined by cytometric bead array (BD Biosciences). ELISA to detect RV antigen in the feces was performed as previously described (23).

Statistics. Mann-Whitney, Wilcoxon signed rank, ANOVA, and 2-tailed $t$ tests were performed using GraphPad Prism (GraphPad Software Inc). $P \leq 0.05$ was considered significant. Error bars indicate SEM.
Study approval. All animal studies were approved by the Stanford Institutional Animal Care Committee. Institutional review board approval was not required for studies involving human cells, as PBMCs were isolated from anonymized leukoreduction chambers obtained from the Stanford Blood Center.

\section{Acknowledgments}

We thank Marco Colonna for the gift of BDCA2-DTR mice. This work was supported by grants from the NIH (R01 AI012362-24 and P30DK56339 to H.B. Greenberg; R01 DK084647 and R37 AI047822 to E.C. Butcher) and VA Merit Review grants to H.B. Greenberg and E.C. Butcher; K. Lahl is supported by the Deutsche Forschungsgemeinschaft (LA 2738/1-1\&2) and a research fellowship (3579) from the Crohn's and Colitis Foundation of America.

Received for publication November 21, 2012, and accepted in revised form February 26, 2013.

Address correspondence to: Harry B. Greenberg, Stanford University School of Medicine, 291 Campus Drive, LKSC Building, Room LK3C02, Stanford, California 94305-5105, USA. Phone: 650.725.9722; Fax: 650.852.3259; E-mail: harry.greenberg@ stanford.edu.

Emily M. Deal's present address is: BD Biosciences, San Jose, California, USA.
1. Macpherson AJ, McCoy KD, Johansen F-E, Brandtzaeg $\mathrm{P}$. The immune geography of IgA induction and function. Mucosal Immunol. 2008;1(1):11-22.

2. Cerutti A, Rescigno M. The biology of intestinal immunoglobulin A responses. Immunity. 2008; 28(6):740-750.

3. Mora J, et al. Generation of gut-homing IgA-secreting B cells by intestinal dendritic cells. Science. 2006; 314(5802):1157-1160.

4. Tezuka H, et al. Regulation of IgA production by naturally occurring TNF/iNOS-producing dendritic cells. Nature. 2007;448(7156):929-933.

5. Xu W, et al. Epithelial cells trigger frontline immunoglobulin class switching through a pathway regulated by the inhibitor SLPI. Nat Immunol. 2007; 8(3):294-303.

6. Strugnell RA, Wijburg OLC. The role of secretory antibodies in infection immunity. Nat Rev Immunol. 2010;8(9):656-667.

7. Wei M, Shinkura R, Doi Y, Maruya M, Fagarasan $\mathrm{S}$, Honjo T. Mice carrying a knock-in mutation of Aicda resulting in a defect in somatic hypermutation have impaired gut homeostasis and compromised mucosal defense. Nat Immunol. 2011; 12(3):264-270.

8. Asselin-Paturel C, et al. Mouse type I IFN-producing cells are immature APCs with plasmacytoid morphology. Nat Immunol. 2001;2(12):1144-1150.

9. Gilliet M, Cao W, Liu YJ. Plasmacytoid dendritic cells: sensing nucleic acids in viral infection and autoimmune diseases. Nat Rev Immunol. 2008;8(8):594-606.

10. Katze MG, He Y, Gale M. Viruses and interferon: a fight for supremacy. Nat Rev Immunol. 2002; 2(9):675-687.

11. Lund JM, Linehan MM, Iijima N, Iwasaki A. Cutting edge: Plasmacytoid dendritic cells provide innate immune protection against mucosal viral infection in situ. J Immunol. 2006;177(11):7510-7514.

12. Swiecki M, Gilfillan S, Vermi W, Wang Y, Colonna M. Plasmacytoid dendritic cell ablation impacts early interferon responses and antiviral NK and CD8(+) T cell accrual. Immunity. 2010;33(6):955-966.

13. Ammann C, Messer R, Peterson K, Hasenkrug K.
Lactate dehydrogenase-elevating virus induces systemic lymphocyte activation via TLR7-dependent IFNalpha responses by plasmacytoid dendritic cells. PLoS One. 2009;4(7):e6105.

14. Smitt J, Rudd B, Lukacs N. Plasmacytoid dendritic cells inhibit pulmonary immunopathology and promote clearance of respiratory syncytial virus. J Exp Med. 2006;203(5):1153-1159.

15. Yoneyama M, et al. Plasmacytoid DCs help lymph node DCs to induce anti-HSV CTLs. JExpMed. 2005; 202(3):425-435.

16. Cerutti A, Qiao X, He B. Plasmacytoid dendritic cells and the regulation of immunoglobulin heavy chain class switching. Immunol Cell Biol. 2005; 83(5):554-562.

17. Jego G, Palucka AK, Blanck J-P, Chalouni C, Pascual V, Banchereau J. Plasmacytoid dendritic cells induce plasma cell differentiation through type I interferon and interleukin 6. Immunity. 2003; $19(2): 225-234$.

18. Le Bon A, Schiavoni G, D’Agostino G, Gresser I, Belardelli F, Tough DF. Type I interferons potently enhance humoral immunity and can promote isotype switching by stimulating dendritic cells in vivo. Immunity. 2001;14(4):461-470.

19. Varani S, et al. Human cytomegalovirus differentially controls B cell and T cell responses through effects on plasmacytoid dendritic cells. J Immunol. 2007;179(11):7767-7776

20. Tezuka H, et al. Prominent role for plasmacytoid dendritic cells in mucosal T cell-independent IgA induction. Immunity. 2011;34(2):247-257.

21. Deal EM, Jaimes MC, Crawford SE, Estes MK, Greenberg HB. Rotavirus structural proteins and dsRNA are required for the human primary plasmacytoid dendritic cell IFNalpha response. PLoS Pathog. 2010;6(6):e1000931.

22 . Sen A, et al. Innate immune response to homologous rotavirus infection in the small intestinal villous epithelium at single-cell resolution. Proc Natl Acad Sci U S A. 2012;109(50):20667-20672.

23. Franco MA, Greenberg HB. Role of B cells and cytotoxic $T$ lymphocytes in clearance of and immu- nity to rotavirus infection in mice. J Virol. 1995; 69(12):7800-7806

24. Jayashree S, Bhan MK, Kumar R, Bhandari N, Sazawal S. Protection against neonatal rotavirus infection by breast milk antibodies and trypsin inhibitors. J Med Virol. 1988;26(3):333-338.

25. Johansen K, Svensson L. Neutralization of rotavirus and recognition of immunologically important epitopes on VP4 and VP7 by human IgA. Arch Virol. 1997;142(7):1491-1498.

26. Ruggeri FM, Greenberg HB. Antibodies to the trypsin cleavage peptide VP8 neutralize rotavirus by inhibiting binding of virions to target cells in culture. J Virol. 1991;65(5):2211-2219.

27. Franco MA, Tin C, Greenberg HB. CD8+ T cells can mediate almost complete short-term and partial long-term immunity to rotavirus in mice.J Virol. 1997;71(5):4165-4170.

28. Marcelin G, Miller AD, Blutt SE, Conner ME. Immune mediators of rotavirus antigenemia clearance in mice. JVirol. 2011;85(15):7937-7941.

29. Kuklin N, et al. Protective intestinal anti-rotavirus B cell immunity is dependent on alpha 4 beta 7 integrin expression but does not require IgA antibody production. J Immunol. 2001;166(3):1894-1902.

30. Narvaez CF, et al. Human rotavirus specific IgM memory B cells have differential cloning efficiency and switch capactiy and play a role in antiviral immunity in vivo. J Virol. 2012;86(19):10829-10840.

31. Blutt SE, Warfield KL, Lewis DE, Conner ME. Early response to rotavirus infection involves massive $\mathrm{B}$ cell activation. J Immunol. 2002;168(11):5716-5721.

32. Blutt SE, Crawford S, Warfield KL, Lewis DE, Estes MK, Conner ME. The VP7 outer capsid protein of rotavirus induces polyclonal B-cell activation. J Virol. 2004;78(13):6974-6981.

33. Wang Y, et al. Rotavirus infection alters peripheral T-cell homeostasis in children with acute diarrhea. J Virol. 2007;81(8):3904-3912.

34. Narvaez CF, Franco MA, Angel J, Morton JM, Greenberg HB. Rotavirus differentially infects and polyclonally stimulates human B cells depending on their differentiation state and tissue of origin. 
J Virol. 2010;84(9):4543-4555

35. Kumagai Y, et al. Alveolar macrophages are the primary interferon-alpha producer in pulmonary infection with RNA viruses. Immunity. 2007; 27(2):240-252

36. Lande R, Gilliet M. Plasmacytoid dendritic cells: key players in the initiation and regulation of immune responses. Ann N Y Acad Sci. 2010;1183:89-103.

37. Siegal FP, et al. The nature of the principal type 1 interferon-producing cells in human blood. Science. 1999;284(5421):1835-1837.

38. Asselin-Paturel C, Brizard G, Pin J-J, Briere F, Trinchieri G. Mouse strain differences in plasmacytoid dendritic cell frequency and function revealed by a novel monoclonal antibody. J Immunol. 2003; 171(12):6466-6477.

39. Feng N, Burns JW, Bracy L, Greenberg HB. Comparison of mucosal and systemic humoral immune responses and subsequent protection in mice orally inoculated with a homologous or a heterologous rotavirus. J Virol. 1994;68(12):7766-7773.

40. Burns JW, Siadat-Pajour M, Krishnaney AA, Greenberg HB. Protective effect of rotavirus VP6-specific IgA monoclonal antibodies that lack neutralizing activity. Science. 1996;272(5258):104-107.

41. Feng N, Vo PT, Chung D, Vo TV, Hoshino Y, Greenberg HB. Heterotypic protection following oral immunization with live heterologous rotaviruses in a mouse model. J Infect Dis. 1997;175(2):330-341.

42. McNeal MM, Barone KS, Rae MN, Ward RL. Effector functions of antibody and CD8+ cells in resolution of rotavirus infection and protection against reinfection in mice. Virology. 1995;214(2):387-397.

43. Blasius AL, Cella M, Maldonado J, Takai T, Colonna M. Siglec-H is an IPC-specific receptor that modulates type I IFN secretion through DAP12. Blood. 2006;107(6):2474-2476.

44. Poeck H, et al. Plasmacytoid dendritic cells, antigen, and CpG-C license human B-cells for plasma cell differentiation and immunoglobulin production in the absence of T-cell help. Blood. 2004 103(8):3058.

45. Bekeredjian-Ding IB, et al. Plasmacytoid dendritic cells control TLR7 sensitivity of naive B-cells via type I IFN. J Immunol. 2005;174(7):4043-4050
46. Green NM, et al. Murine B cell response to TLR7 ligands depends on an IFN- $\beta$ feedback loop. J Immunol. 2009;183(3):1569-1576.

47. Thibault DL, Graham KL, Lee LY, Balboni I, Hertzog PJ, Utz PJ. Type I interferon receptor controls $B$ cell expression of nucleic acid-sensing Tolllike receptors and autoantibody production in a murine model of lupus. Arthritis Res Ther. 2009; 11(4):R112.

48. Chang WL, Coro ES, Rau FC, Xiao Y, Erle DJ, Baumgarth N. Influenza virus infection causes global respiratory tract B cell response modulation via innate immune signals. J Immunol. 2007; 178(3):1457-1467.

49. Coro ES, Chang WL, Baumgarth N. Type I IFN receptor signals directly stimulate local B cells early following influenza virus infection. J Immunol. 2006;176(7):4343-4351

50. Purtha WE, Chachu KA, Virgin HW 4th, Diamond MS. Early B-cell activation after West Nile virus infection requires alpha/beta interferon but not antigen receptor signaling. J Virol. 2008; 82(22):10964-10974

51 . Woods A, et al. Influenza virus-induced type I interferon leads to polyclonal B-cell activation but does not break down B-cell tolerance. J Virol. 2007; 81(22):12525-12534

52. Burns JW, Krishnaney AA, Vo PT, Rouse RV, Anderson LJ, Greenberg HB. Analyses of homologous rotavirus infections in the mouse model. Virology. 1995; 207(1):143-153.

53. Zhang J, et al. Characterization of Siglec-H as a novel endocytic receptor expressed on murine plasmacytoid dendritic cell precursors. Blood. 2006; 107(9):3600-3608.

54. Bernstein DI, Ziegler JM, Ward RL. Rotavirus fecal $\operatorname{IgA}$ antibody response in adults challenged with human rotavirus. J Med Virol. 1986;20(4):297-304.

55. O'Neal CM, Harriman GR, Conner ME. Protection of the villus epithelial cells of the small intestine from rotavirus infection does not require immunoglobulin A. J Virol. 2000;74(9):4102-4109.

56. Ruggeri FM, Johansen K, Basile G, Kraehenbuhl J-P, Svensson L. Antirotavirus immunoglobulin a neutralizes virus in vitro after transcytosis through epithelial cells and protects infant mice from diarrhea. J Virol. 1998;72(4):2708-2714

57. Corthesy B, et al. Rotavirus anti-VP6 secretory immunoglobulin A contributes to protection via intracellular neutralization but not via immune exclusion. J Virol. 2006;80(21):10692-10699.

58. Feng $\mathrm{N}$, et al. Inhibition of rotavirus replication by a non-neutralizing, rotavirus $\mathrm{VP}$-specific IgA mAb. J Clin Invest. 2002;109(9):1203-1213.

59. Velazquez FR, et al. Serum antibody as a marker of protection against natural rotavirus infection and disease. J Infect Dis. 2000;182(6):1602-1609.

60. Heer AK, et al. TLR signaling fine-tunes anti-influenza $B$ cell responses without regulating effector $T$ cell responses. J Immunol. 2007;178(4):2182-2191.

61. Zhu J, Huang X, Yang Y. Type I IFN signaling on both $\mathrm{B}$ and CD4 $\mathrm{T}$ cells is required for protective antibody response to adenovirus. J Immunol. 2007; 178(6):3505-3510.

62. Contractor N, Louten J, Kim L, Biron CA, Kelsall BL. Cutting edge: Peyer's patch plasmacytoid dendritic cells ( $\mathrm{pDCs}$ ) produce low levels of type I interferons: possible role for IL-10, TGFbeta, and prostaglandin E2 in conditioning a unique mucosal pDC phenotype. J Immunol. 2007;179(5):2690-2694.

63. Fenaux M, Cuadras MA, Feng N, Jaimes M, Greenberg HB. Extraintestinal spread and replication of a homologous EC rotavirus strain and a heterologous rhesus rotavirus in BALB/c mice. JVirol. 2006; 80(11):5219-5232.

64. Cuadras MA, Feigelstock DA, An S, Greenberg HB. Gene expression pattern in Caco-2 cells following rotavirus infection. J Virol. 2002;76(9):4467-4482.

65. Hoshino Y, Wyatt RG, Greenberg HB, Flores J, Kapikian AZ. Serotypic similarity and diversity of rotaviruses of mammalian and avian origin as studied by plaque-reduction neutralization.J Infect Dis. 1984;149(5):694-702

66. Wei C, Jung J, Sanz I. OMIP-003: phenotypic analysis of human memory B cells. Cytometry A. 2011; 79(11):894-896

67. Warfield KL, et al. Induction of humoral and CD8+ $\mathrm{T}$ cell responses are required for protection against lethal Ebola virus infection. J Immunol. 2005; 175(2):1184-1191. 